\title{
Simulation studies of the expected proton rejection capabilities of CALET
}

\section{Francesco Palma for the CALET collaboration}

University of Rome Tor Vergata and INFN, Italy

E-mail: francesco.palmalroma2.infn.it

The CALorimetric Electron Telescope (CALET) is a Japanese led international space mission by JAXA (Japanese AeroSpace Agency) in collaboration with the Italian Space Agency (ASI) and NASA. The instrument will be launched to the International Space Station (ISS) in 2015.

The major scientific goals for CALET are to measure the flux of cosmic-ray electrons (including positrons) from $1 \mathrm{GeV}$ to $20 \mathrm{TeV}$, gamma rays to $10 \mathrm{TeV}$ and nuclei from $\mathrm{Z}=1$ to 40 up to $1000 \mathrm{TeV}$. These measurements are essential to search for dark matter signatures, investigate the mechanism of cosmic-ray acceleration and propagation in the Galaxy and discover possible astrophysical sources of high-energy electrons nearby the Earth.

The instrument consists of two layers of segmented plastic scintillators for the cosmic-ray charge identification, a 3 radiation length thick tungsten-scintillating fiber imaging calorimeter and a 27 radiation length thick lead-tungstate calorimeter.

Protons are the largest source of background for the high-energy electron observation. As the ratio of protons to electrons increases at higher energies, a proton rejection power better than $10^{5}$ is necessary to measure the electron spectrum with a proton contamination below a few percent in the $\mathrm{TeV}$ region.

In this work, a Monte Carlo based study of the proton rejection capability CALET can achieve at $\mathrm{TeV}$ energies is presented. Both standard analysis based on consecutive selection criteria and multivariate analysis are applied to simulated samples of signal and background events.

The 34th International Cosmic Ray Conference,

30 July- 6 August, 2015

The Hague, The Netherlands 


\section{Introduction}

CALET (CALorimetric Electron Telescope) [1] is a Japanese-led international mission funded by the Japanese Space Agency (JAXA), the Italian Space Agency (ASI) and NASA. The instrument was designed and built in Japan, with hardware contributions from Italy and assistance from collaborators in Italy and the United States. The instrument will be launched in 2015 by a Japanese carrier, H-II Transfer Vehicle (HTV), and robotically installed on the Japanese Experiment ModuleExposed Facility (JEM-EF) on the International Space Station (ISS) for a 5 year mission collecting new data on high-energy cosmic and gamma rays. The primary science goal of CALET is to perform high-precision measurements of the electron spectrum from $1 \mathrm{GeV}$ to $20 \mathrm{TeV}$ in order to observe discrete sources of high-energy particle acceleration in our local region of the Galaxy. Thanks to its observations of cosmic-ray electrons and gamma rays from a few $\mathrm{GeV}$ up to 10 - 20 $\mathrm{TeV}$ and nuclei from a few $10 \mathrm{GeV}$ up to the $1000 \mathrm{TeV}$ range, the CALET mission will address many of the outstanding questions of High-Energy Astroparticle Physics, such as the origin of cosmic rays (CRs), the mechanism of $\mathrm{CR}$ acceleration and galactic propagation, the existence of dark matter and nearby CR sources. It will also monitor gamma-ray transients with a dedicated gamma-ray burst instrument and study solar modulation. The left panel of Fig. 1 shows a schematic overview of the CALET instrument with the main calorimeter and Gamma-Ray Burst Monitor subsystems.
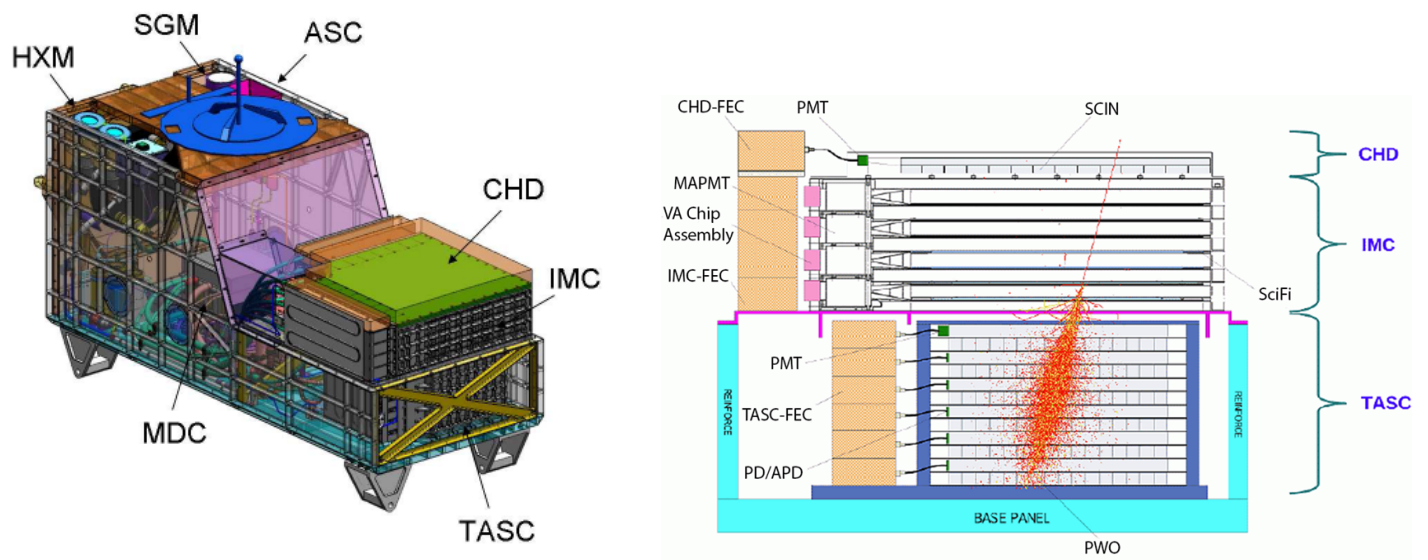

Figure 1: (Left panel) CALET instrument package showing the main calorimeter and Gamma-Ray Burst Monitor sub-systems. (Right panel) Schematic view of CALET. The picture of a simulated $1 \mathrm{TeV}$ electron is superimposed.

\section{The CALET instrument}

Heart of the mission is the main telescope, shown in detail in the right panel of Fig. 1, which has a field of view of $\sim 45^{\circ}$ from the zenith. CALET is an all-calorimetric instrument, with a total thickness equivalent to 30 radiation lengths $\left(\mathrm{X}_{0}\right)$ and 1.3 proton interaction lengths $\left(\lambda_{I}\right)$, preceded by a particle identification system. The energy measurement relies on two kinds of calorimeters: a fine grained pre-shower, known as IMaging Calorimeter (IMC), followed by a 
Total AbSorption Calorimeter (TASC). The effective geometrical factor of CALET for high-energy electrons is $\simeq 1200 \mathrm{~cm}^{2}$ sr and the total weight of the system will be approximately $650 \mathrm{~kg}$. In order to identify individual chemical elements in the cosmic-ray flux, a CHarge Detector (CHD) has been designed to measure the charge of the incoming nuclei via the $Z^{2}$ dependence of the specific ionization loss in a double layered, segmented, plastic scintillator array positioned above the IMC. Each layer is made of 14 plastic scintillator paddles, with dimensions $45 \mathrm{~cm}(\mathrm{~L}) \times 3.2$ $\mathrm{cm}(\mathrm{W}) \times 1 \mathrm{~cm}(\mathrm{H})$. This segmented configuration has been optimized to reduce multi-hits on each paddle caused by backscattering particles. The two layers of paddles are orthogonally arranged to determine the incident position of cosmic rays. Scintillation light from each paddle is collected and readout by a photomultiplier tube (PMT). The CHD and related front-end electronics have been designed to provide incident particle identification over a large dynamic range for charges from $Z=1$ to $Z=40$ [2]. The IMC will image the early shower profile with a fine granularity by using $1 \mathrm{~mm}^{2}$ squared cross-section scintillating fibers ( $\mathrm{SciFi}$ ) individually readout by Multi-Anode PhotoMultiplier Tubes (MAPMTs). The imaging pre-shower consists of 7 layers of tungsten plates, each separated by 2 layers of scintillating fibers, arranged in belts along the $\mathrm{X}$ and $\mathrm{Y}$ directions, and is capped by an additional X,Y SciFi layer pair. Each SciFi layer is assembled with 448 fibers and its dimensions are $44.8 \mathrm{~cm}(\mathrm{~L}) \times 44.8 \mathrm{~cm}(\mathrm{~W}) \times 0.2 \mathrm{~cm}(\mathrm{H})$. The total thickness of the IMC is equivalent to $3 \mathrm{X}_{0}$. The first five tungsten-SciFi layers sample the shower every $0.2 \mathrm{X}_{0}$ while the last two provide $1.0 \mathrm{X}_{0}$ sampling. The IMC fine granularity allows to: (i) reconstruct the incident particle trajectory [3]; (ii) determine the starting point of the shower; (iii) separate the incident from backscattered particles. The SciFi layers are readout by 112 64-anode Hamamatsu R7600M64 photomultiplier tubes (MAPMTs). The homogeneous calorimeter is designed to measure the total energy of the incident particle and discriminate electromagnetic from hadronic showers. TASC is composed of 12 layers, each comprised of 16 lead tungstate (PWO) logs. Each log has dimensions of $326 \mathrm{~mm}(\mathrm{~L}) \times 19 \mathrm{~mm}(\mathrm{~W}) \times 20 \mathrm{~mm}(\mathrm{H})$. Layers are alternately arranged with the logs oriented along orthogonal directions to provide a 3D reconstruction of the showers. Six layers image the $\mathrm{XZ}$ view and 6 the $\mathrm{YZ}$ view. The surface of each layer is $991 \mathrm{~cm}^{2}$ and the total TASC thickness corresponds to about $27 \mathrm{X}_{0}$ and 1.2 $\lambda_{I}$ at normal incidence. Each PWO log in the upper layer (16 bars) is readout by a PMT to generate a trigger signal. Hybrid packages of silicon Avalanche PhotoDiode and silicon PhotoDiode (Dual APD/PD) are used to collect light from the remaining PWO bars. According to Monte Carlo simulations and beam test data, TASC can measure the energy of the incident electrons and gamma rays with resolution $\sim 2 \%$ above 100 $\mathrm{GeV}$.

\section{The Monte Carlo simulation}

Samples of signal (electron) and background (proton) events are generated by means of a detailed simulation of the CALET instrument based on Monte Carlo package EPICS (ElectronPhoton Induced Cascade Simulator in a detector) [4]. DPMJET-III [5] is adopted as a hadronic interaction model. Incident particles are generated isotropically on a $78 \mathrm{~cm}$ radius hemisphere with its center on the top of TASC. Events for the analysis are preliminary selected by requiring that the generated particles cross the 4th IMC layer and the top TASC layer, and their pathlength inside the TASC is longer than $10 \mathrm{X}_{0}$. Preselected events are then classified into four acceptance 
categories, depending on the direction of the incident particle. In Type 1 acceptance, the incident particle direction crosses the first IMC layer, the top and bottom TASC layers, and is well contained within a fiducial volume in the TASC (defined by excluding the logs at the TASC edges in each layer). Conversely, in Type 2 acceptance, the primary particle direction is outside the TASC fiducial volume. Events, whose direction crosses the 4th IMC SciFi layer, the top and bottom TASC layers, belong to Type 3 acceptance. Finally, if the incident particle direction crosses the 4th IMC layer, the top layer and one lateral side of the TASC, the event is classified into the 4th acceptance category. In order to estimate the proton rejection power at $1 \mathrm{TeV}$, the following samples are simulated: $\sim 1.1 \times 10^{6}$ proton events inside acceptance with energies $1 \mathrm{TeV}<E<100 \mathrm{TeV}$ and $\sim 1.74 \times 10^{5}$ electrons inside acceptance with $20 \mathrm{GeV}<E<2000 \mathrm{GeV}$. Both samples are generated according to a power-law distribution $d N / d E \propto E^{-\gamma}$, where $\gamma=1.0$, in order to collect enough events in high-energy bins.

\section{Selection criteria for electron/proton separation}

TASC will measure the development of the electromagnetic/hadronic shower in order to determine the total energy of the incident particle. Protons may constitute a potential source of contamination for the detection of $1 \mathrm{TeV}$ electrons if they release energy deposits in the TASC in the same range as $1 \mathrm{TeV}$ electrons. In this analysis, the proton rejection power is studied in a bin of energy deposited in the TASC, $912 \mathrm{GeV}<E_{\text {TASC }}<1000 \mathrm{GeV}$, whose width is chosen according to the expected electron statistics. The proton events, whose energy deposits are inside the reference energy bin, have incident energies $1 \mathrm{TeV} \leq E \leq 15 \mathrm{TeV}$. This is due to the lower resolution of the TASC for hadronic showers compared to electrons ( $\sim 40 \%$ for $1 \mathrm{TeV}$ protons). Only $1.5 \%$ out of $\sim 10^{6}$ events inside acceptance releases energy deposits inside the reference bin and may contaminate the electron sample ( $\sim 3400$ events). It is clear that the different instrument energy response to protons and electrons significantly contributes to the proton rejection power. Another efficient contribution to the proton rejection power comes from applying the High-Energy Trigger, HET. The signal from the top TASC layer and the sum of the signals from the 7th and 8th IMC layers are used to select events mainly interacting in the $3 \mathrm{X}_{0}$ thick IMC (electrons) and reject events interacting deep in the TASC (protons). The HET rejects about 55\% of the proton events that would release TASC energy deposits in the bin $912 \mathrm{GeV}<E_{T A S C}<1000 \mathrm{GeV}$, while retaining almost all the electrons. In the following sections, some selection criteria, as already used in a previous analysis by [6], will be introduced in order to further discriminate electrons against background events on the basis of their different shower development in the TASC. These criteria will be applied on the sub-samples of HE-triggered events.

\section{1 $f_{E} v s . R_{E}$ cut}

In a calorimetric instrument such as CALET, electron/proton $(e / p)$ separation relies strongly on the shower development profile. A variable which gives a quantitative description of the different longitudinal shower development in the calorimeter between electrons and protons is the fractional energy deposited in the last hit TASC layer: $f_{E}=E_{\text {exit layer }} / E_{T A S C}$, where $E_{\text {exit layer }}$ is the energy deposited in the layer where the incident particle axis leaves off the TASC. $f_{E}$ variable alone provides quite good $e / p$ discrimination for events impinging on the TASC at 
high zenith angle (Type 3 and 4 acceptance), i.e. with a large pathlength in the calorimeter. For events with lower pathlength, it is necessary to introduce another discriminant variable, the energy weighted spread, that is a measure of the lateral width of the shower in the TASC and is defined as $R_{E}=\sqrt{\sum_{i}\left(\sum_{j} \Delta E_{i j} \times R_{i}^{2}\right) / \sum_{i} \sum_{j} \Delta E_{i j}}$, where $\Delta E_{i j}$ is the energy deposited in the $j$ th PWO crystal in the $i$ th layer. The root mean square spread $R_{i}$ at the $i$ th layer $(0 \leq i \leq 11)$ is given by $R_{i}=\sqrt{\sum_{j}\left(\Delta E_{i j} \times\left(x_{i j}-x_{i c}\right)^{2}\right) / \sum_{j} \Delta E_{i j}}$, where $x_{i c}$ is the coordinate of the intercept of the incident particle direction with the $i$ th layer, and $x_{i j}$ is the coordinate of the center of the $j$ th PWO crystal in the $i$ th layer. The variables $f_{E}$ and $R_{E}$ are calculated for each HE-triggered event with an energy deposit in the reference bin $(912$ - 1000) $\mathrm{GeV}$ and plotted in a plane, where a two dimensional cut is applied separately for each acceptance category (Fig. 2). The events in the region below the black line can be selected as electrons. However, a few proton events within the electron region (28 out of the 7821 HE-triggered events) still survive.

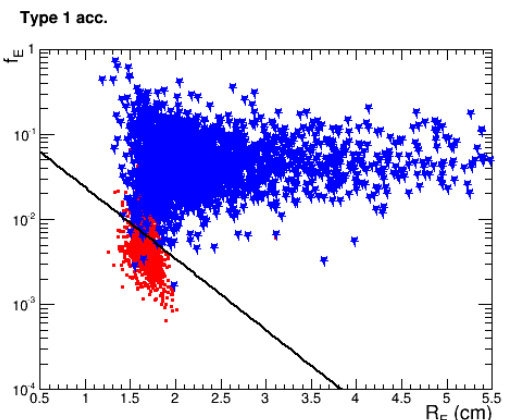

Type 3 acc.

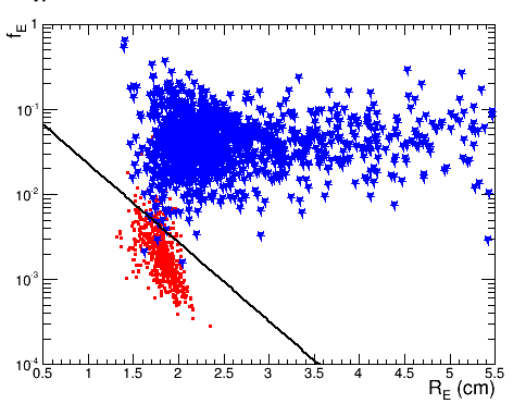

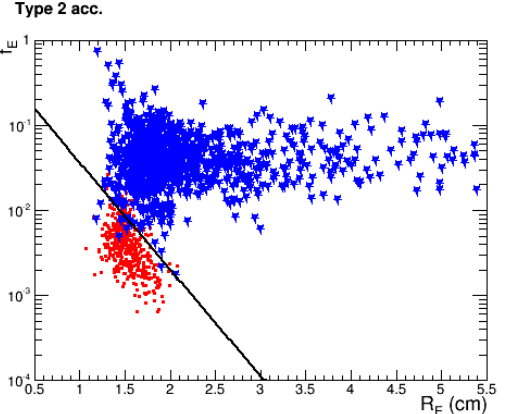

Type 4 acc

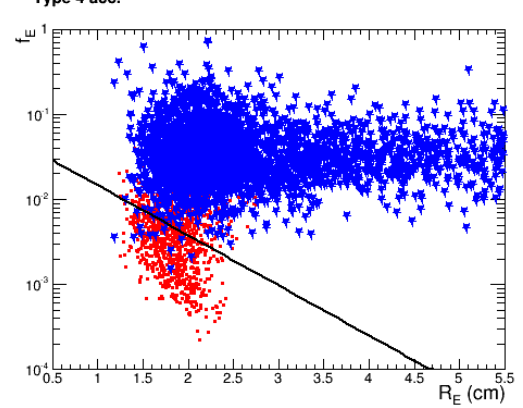

Figure 2: $f_{E} v s . R_{E}$ for electrons (red points) and protons (blue star points) in the four acceptance categories. The black line represents the selection cuts applied in each case.

\subsection{The IMC and CHD cuts}

In order to discard the proton events surviving the $f_{E} v s . R_{E}$ cut, two additional selection cuts are applied with respect to [6] and are based on the following variables: the ratio $E_{1 M R} / E_{I M C}$ between the energy deposited in the last IMC layer within 1 Molière radius from the primary particle direction and the total energy deposited in the IMC, and the total energy $E_{C H D}$ deposited in the CHD paddles (Fig. 3). These selection cuts are useful to reject protons interacting very early in the instrument, in the IMC or even in the CHD. These proton events may induce showers with similar characteristics to electromagnetic ones and are therefore difficult to be rejected by the 
$f_{E} v s . R_{E}$ cut. After the cuts $E_{1 M R} / E_{I M C}>0.455$ and $E_{C H D}<0.2 \mathrm{GeV}$ are applied in sequence, the number of contaminating protons amounts to 7 events.
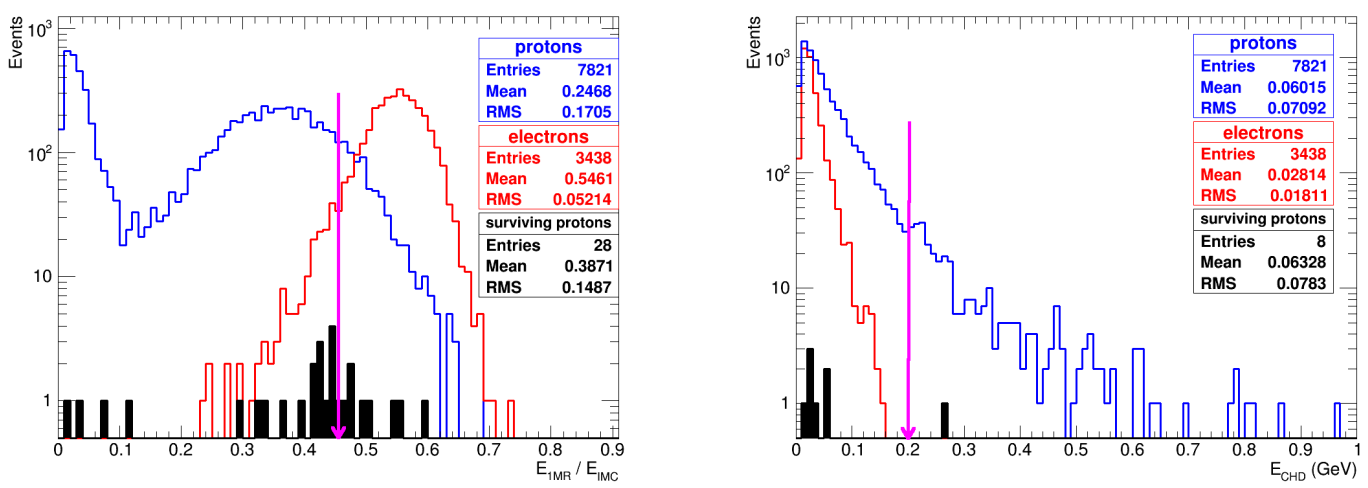

Figure 3: (Left panel) Distribution of $E_{1 M R} / E_{I M C}$ for electrons (red) and protons (blue). The applied cut value $\left(E_{1 M R} / E_{I M C}>0.455\right)$ is shown by the arrow. (Right panel) Distribution of the integrated CHD paddle signal for electrons (red) and protons (blue). The applied cut value $\left(E_{C H D}<0.2 \mathrm{GeV}\right)$ is shown by the arrow. The black histogram in the left panel corresponds to the 28 proton events selected by the $f_{E} v s . R_{E}$ cut, while the black one in the right panel contains the 8 proton events surviving the IMC cut.

\section{Electron/proton separation}

The numbers of electrons and protons selected by each single cut are summarized for each acceptance category in Table 1 . The cuts are applied in sequence from top to bottom. The final number of protons surviving all the selection criteria is 7 out of the initial $\sim 1.1 \times 10^{6}$ events. This method allows to achieve an overall electron efficiency $\varepsilon_{c, e}=(70.0 \pm 0.8) \times 10^{-2}$, a proton efficiency $\varepsilon_{c, p}=\left(6.2_{-2.3}^{+3.4}\right) \times 10^{-6}$ and confirms a proton rejection power in the $\mathrm{TeV}$ energy region of the order of $10^{5}, R=\varepsilon_{c, e} / \varepsilon_{c, p}=\left(1.1_{-0.4}^{+0.6}\right) \times 10^{5}$. The upper and lower limits for the values of the proton efficiency are calculated assuming a Poisson statistics for the small number of residual according to [7]. Though these results have been obtained with $E^{-1}$ generation spectrum, a comparable $e / p$ separation is expected when simulating realistic power-law spectra for both electrons and protons. The irreducible hadronic background is likely caused by protons producing at least one high-energy neutral pion in the upper part of the detector. Neutral pions may in turn induce "electron-like" showers in the TASC that are indistinguishable from electron-induced showers.

\section{Multivariate analysis}

Multivariate analysis (MVA) is adopted as an alternative method to evaluate CALET capability to discriminate electrons against protons. In particular the Toolkit for Multivariate Data Analysis with ROOT (TMVA) [8] is used for the present work. TMVA provides different classifier methods, both linear and non-linear, to select signal events from a sample of background events. Different multivariate methods have been trained with the same electron and proton samples used in the 


\begin{tabular}{cccccc}
\hline \hline Protons & \multicolumn{5}{c}{} \\
\hline \hline Cuts & Type 1 acc. & Type 2 acc. & Type 3 acc. & Type 4 acc. & Total \\
\hline Inside acceptance & 422168 & 155212 & 194355 & 353075 & 1124810 \\
Energy bin & 6333 & 2394 & 3151 & 5422 & 17300 \\
HET & 2600 & 1019 & 1319 & 2883 & 7821 \\
$f_{E}$ vs. $R_{E}$ & 5 & 7 & 3 & 13 & 28 \\
$E_{1 M R} / E_{I M C}>0.455$ & 3 & 2 & 0 & 3 & 8 \\
$E_{C H D}<0.2 \mathrm{GeV}$ & 3 & 1 & 0 & 3 & 7 \\
\hline \hline Electrons & & & & & \\
\hline \hline Cuts & Type 1 acc. & Type 2 acc. & Type 3 acc. & Type 4 acc. & Total \\
\hline Inside acceptance & 65525 & 24239 & 30319 & 54345 & 174428 \\
Energy bin & 1321 & 467 & 569 & 1081 & 3438 \\
HET & 1321 & 467 & 569 & 1081 & 3438 \\
$f_{E} v s . R_{E}$ & 966 & 418 & 502 & 622 & 2508 \\
$E_{1 M R} / E_{I M C}>0.455$ & 937 & 400 & 476 & 594 & 2407 \\
$E_{C H D}<0.2 \mathrm{GeV}$ & 937 & 400 & 476 & 594 & 2407 \\
\hline \hline
\end{tabular}

Table 1: The number of electrons and protons selected by each cut used for $e / p$ discrimination in the TeV energy region. The cuts are applied in sequence from top to bottom.

previous analysis and Boosted Decision Trees (BDT) turned out to be the most performing for the present classification problem. Eleven variables have been used as input to train each MVA method. In addition to the already described variables, $R_{E}, f_{E}, E_{1 M R} / E_{I M C}$ and $E_{C H D}$, the following variables were used:

- the maximum depth of the shower $t_{\max }=\alpha / b, b, \chi^{2} / n d f$ and the starting point of the shower as obtained by fitting the longitudinal shower profile imaged by TASC to the function $\frac{d E}{d t}=$ $E_{0} \frac{b^{(\alpha+1)}}{\Gamma(\alpha+1)} t^{\alpha} e^{-b t}$, where $t=x / X_{0}$ is the longitudinal depth in units of radiation length $X_{0}$ and $E_{0}$ is the energy of the incident particle;

- the parameters $p_{0}, p_{1}$ and $\chi^{2} / n d f$ as obtained by fitting the longitudinal shower profile imaged by IMC to a parabola, $d E / d t=p_{0} t^{2}+p_{1}$, where $t$ is the longitudinal depth.

The trained MVA method is then applied to the test data set and provides scalar outputs according to which an event can be classified as either signal or background. In this analysis, the samples of HE-triggered events are split in half for training and half for testing (1719 electrons and about 3870 protons). Fig. 4 shows the BDT output distributions for signal and background events from the test sample. By applying the cut BDT response $>0.315$ (black arrow in Fig. 4) to the test sample, efficiencies of $\sim 82 \%$ and $\sim 6.22 \times 10^{-6}$ for electrons and protons are found, respectively, corresponding to a proton rejection power $\sim 1.32 \times 10^{5}$.

\section{Conclusions}

A Monte Carlo based study is performed to estimate the $e / p$ discrimination CALET can 


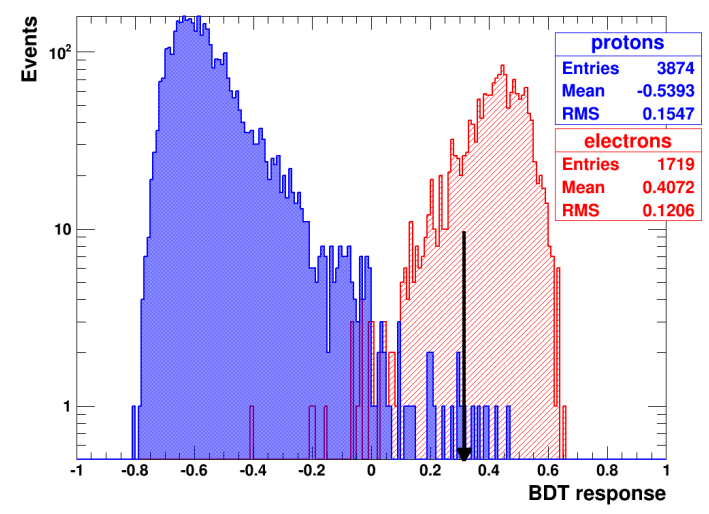

Figure 4: BDT output distributions for electron (red) and proton (blue) events from the test sample. The applied cut value $(B D T$ response $>0.315)$ is shown by the black arrow.

achieve at $\mathrm{TeV}$ energies. For this purpose, selection criteria based on the shower shape analysis of events in the TASC are developed and applied in sequence to Monte Carlo simulated samples of signal and background events. Information from CHD and IMC is further used to improve the $e / p$ discrimination and reduce the residual proton background. An overall proton rejection power $\left(1.1_{-0.4}^{+0.6}\right) \times 10^{5}$ is estimated for the electron energy range $912-1000 \mathrm{GeV}$ with EPICS simulation, with a proton efficiency $\left(6.2_{-2.3}^{+3.4}\right) \times 10^{-6}$ and an electron efficiency $(70.0 \pm 0.8) \%$. These results have also been compared to those obtained from multivariate analysis. With equal values for proton efficiency $\sim 6.22 \times 10^{-6}$ and rejection power $\sim 1.32 \times 10^{5}$, the analysis with BDT shows an increased electron efficiency $\sim 82 \%$ compared to the analysis based on consecutive selection cuts. According to the present study, CALET is shown to be able to perform a measurement of the cosmic electron spectrum with a proton contamination below a few percent at $\mathrm{TeV}$ energies where the proton intensity is $10^{3}$ higher than the electron one. CALET measurements will thus contribute to investigate possible nearby astrophysical sources of high-energy electrons and search for dark matter signatures.

\section{References}

[1] S. Torii for the CALET collaboration, Proc. of the 34th ICRC (The Hague), (2015).

[2] P. S. Marrocchesi et al., NIM A 659, 477-483 (2011).

[3] P. Brogi et al. for the CALET collaboration, Proc. of the 34th ICRC (The Hague), ID:510 (2015).

[4] EPICS web page. "EPICS web page”. http://cosmos.n.kanagawa-u.ac.jp/EPICShome/.

[5] DPMJET-III web page. "DPMJET-III web page”. http://sroesler.web.cern.ch/sroesler/dpmjet3.html.

[6] Y. Akaike for the CALET collaboration, Proc. of the 32nd ICRC (Beijing), Vol.6 346 (2011).

[7] N. Gehrels, Astrophysical Journal 303, 336-346 (1986).

[8] A. Hoecker, P. Speckmayer, J. Stelzer, J. Therhaag, E. von Toerne, and H. Voss, "TMVA: Toolkit for Multivariate Data Analysis," PoS A CAT 040 (2007) [physics/0703039]. 